\title{
Ground clutter cancellation in incoherent radars: solutions for EISCAT Svalbard radar
}

\author{
T. Turunen ${ }^{1}$, J. Markkanen ${ }^{2}$, A. P. van Eyken ${ }^{3}$ \\ ${ }^{1}$ EISCAT Scientific Association, SE-98123 Kiruna, Sweden \\ 2 EISCAT Scientific Association, FIN-99600 Sodankylä, Finland \\ ${ }^{3}$ EISCAT Scientific Association, N-9171 Longyearbyen, Norway
}

Received: 24 January 2000 / Revised: 5 July 2000 / Accepted: 6 July 2000

\begin{abstract}
Incoherent scatter radars measure ionosphere parameters using modified Thomson scatter from free electrons in the target (see e.g. Hagfors, 1997). The integrated cross section of the ionospheric scatterers is extremely small and the measurements can easily be disturbed by signals returned by unwanted targets. Ground clutter signals, entering via the antenna side lobes, can render measurements at the nearest target ranges totally impossible. The EISCAT Svalbard Radar (ESR), which started measurements in 1996, suffers from severe ground clutter and the ionosphere cannot be measured in any simple manner at ranges less than about $120-150 \mathrm{~km}$, depending on the modulation employed. If the target and clutter signals have different, and clearly identifiable, properties then, in principle, there are always ways to eliminate the clutter. In incoherent scatter measurements, differences in the coherence times of the wanted and unwanted signals can be used for clutter cancellation. The clutter cancellation must be applied to all modulations, usually alternating codes in modern experiments, used for shorter ranges. Excellent results have been obtained at the ESR using a simple pulse-to-pulse clutter subtraction method, but there are also other possibilities.
\end{abstract}

Key words: Radio science (ionospheric physics; signal processing; instruments and techniques)

\section{Introduction}

Incoherent scatter radars have been designed to study the Earth's ionosphere in the altitude range from some tens of kilometres to several thousand kilometres. The physical mechanism is scattering from free electrons moving under the influence of the much heavier ionospheric ions. The ionosphere is a distributed target, with very small integrated target cross sections, and very

Correspondence to: A. P. van Eyken

e-mail: tony@eiscat.uit.no powerful and sensitive systems must be deployed to ensure useable returns. Incoherent scatter radars typically use VHF and UHF frequencies through several MF instruments also exist.

To obtain adequate spatial and spectral resolution, on-off amplitude modulation, binary phase modulation, frequency modulation, and combinations of these are used in the transmitted pulses. Target bandwidths vary from hundreds of Hertz to some tens of kiloHertz and modulation bandwidths from about one kiloHertz to hundreds of kiloHertz. Together, these dictate the receiving bandwidth, noise bandwidth and sampling intervals required. In some special applications much higher bandwidths must be accommodated. Reception and data sampling usually start 300-500 microseconds after the end of transmitted pulse, for those modulations to be used at the lowest ranges of the target. The upper limit of the possible reception window is set by the point at which the transmitted modulation is no longer useful for the target measurements or by the point where the signal to noise ratio become too small.

In incoherent scatter experiments all the necessary real time computations can be performed in a modulation independent manner by computing a lag profile matrix as described by Turunen and Silen (1984) and Turunen (1986). Lehtinen and Häggström (1987) introduced a family of modulation schemes called alternating codes, where the processing requires the computation of a separate lag profile matrix for every individual modulation, followed by (FIR) filtering of the individual matrix diagonals using code dependent coefficients. These now form the basis of most measurements at the EISCAT radars.

The final limiting factor in incoherent scatter experiments is the thermal noise level and even very low-level unwanted signals can severely disrupt the measurements. There are two main sources of disturbing signals: satellite echoes and ground clutter. Satellite echoes are transient phenomena and can be eliminated by rejecting the parts of data where echoes are present. Where present, ground clutter is a permanent feature and represents a severe problem in the radar system. 
Clutter arises both from the ground itself and from individual targets on the ground and in the air and can also be generated by waves in the ocean, turbulence in the atmosphere, etc. The total received clutter is a linear combination of signals arising from a large number of reflecting bodies at different ranges and of different cross section. For practical antennas, clutter enters the radar system through the side lobes of the radiation pattern at all azimuths and elevations and the dynamic amplitude range of the clutter signal as a function of target distance can cover several orders of magnitude. Over short time scales, the clutter signals are not random but at any given propagation delay they are essentially stationary deterministic signals with constant amplitude and phase.

The most effective way to eliminate such clutter is to build the radar in a valley with no line of sight directions to possible cluttering targets at distances approaching the ranges of the wanted target. This has been achieved for the EISCAT Tromsø radars (Folkestad et al., 1983) but the EISCAT Svalbard Radar (Wannberg et al., 1997) is located on a mountain and has cluttering targets at all ranges up to about $110 \mathrm{~km}$ (see Fig. 1 and EISCAT Annual Report 1996-1997, p. 12).

In the following we assume that the radar system remains linear at all times while receiving, the radar transmitter and receiver maintain phase coherence, and the transmitted power is nearly constant from pulse to pulse (all valid assumptions for almost all operations at the ESR). If these conditions are valid, the phase and amplitude of the clutter signal remain essentially constant from pulse to pulse at any given propagation delay as long as the transmitted modulation remains unchanged. In practice, the clutter pattern changes slowly because the refraction coefficient of the atmosphere changes, the clutter may contain elements from moving targets, and there may be technical weaknesses in the system. However, for the time scales of interest, i.e. over some tens of milliseconds, the clutter pattern can be treated as effectively constant.

Incoherent scatter experiments produce target information only as expectation values. For effective clutter removal, it is enough that the residual clutter component after the primary clutter cancellation is small, and that the event to event distribution of this component has zero mean in every lag profile matrix element needed to be computed in the subsequent signal processing.

There are several possible methods by which nearconstant clutter can be removed. Examples include: subtraction of a constant clutter profile obtained by integration in the amplitude domain, subtraction of a pre-determined clutter profile in the power domain, and pulse-to-pulse subtraction in the amplitude domain. It is also possible to imagine more complicated methods such as fitting a low order curve to the temporal behaviour of the amplitude domain estimates at each lag. However, methods requiring extensive computational resources may have practical limitations, especially in on-line use.

In the next two sections, we discuss three different clutter cancellation methods for incoherent scatter experiments including a well-tested and efficient pulseto-pulse subtraction method implemented at the EISCAT Svalbard Radar.

\section{Some possibilities for the removal of the ground clutter components from incoherent scatter data}

Ground clutter cancellation is a filtering operation which can be performed either in the complex amplitude domain or in the power domain. The filtering may

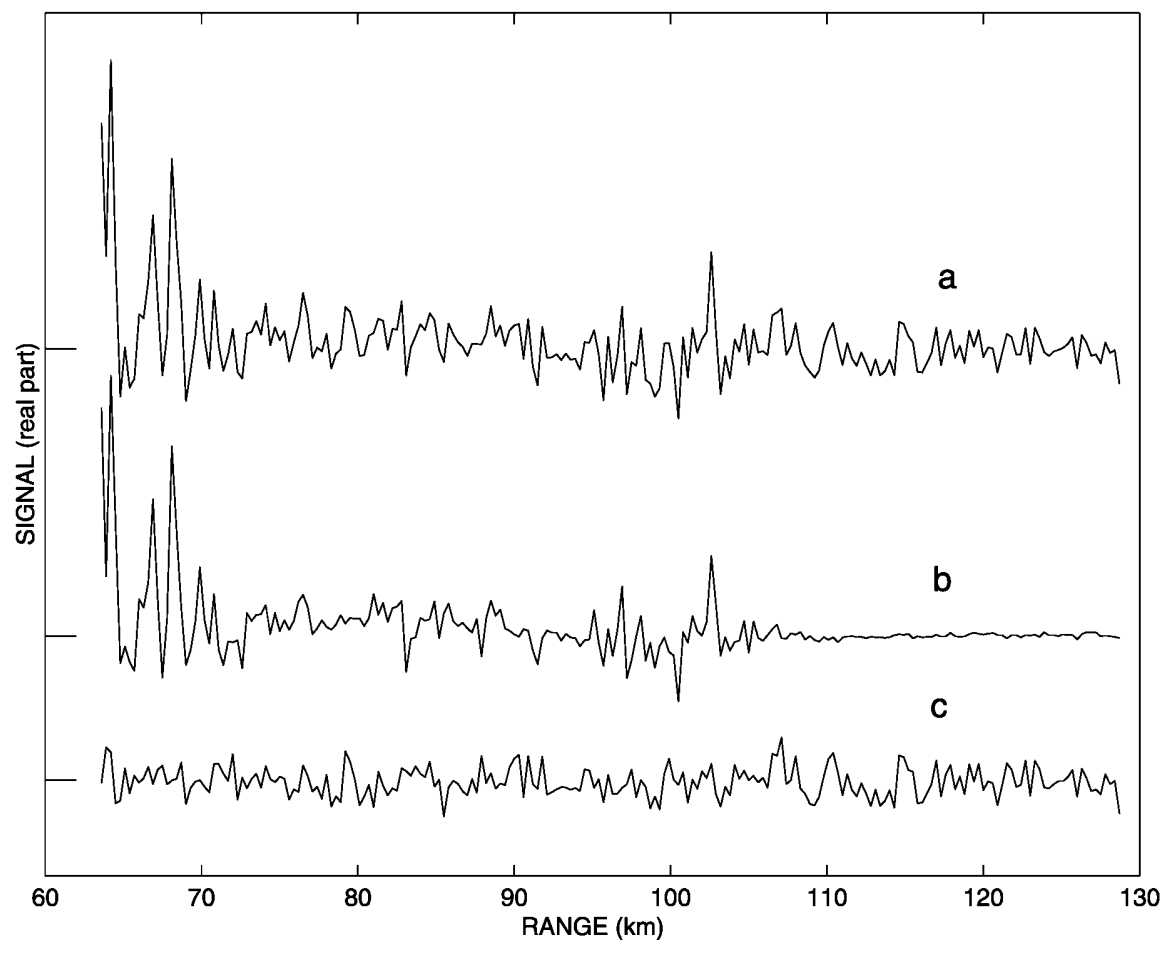

Fig. 1. ESR clutter subtraction using an integrated clutter profile. The ground clutter diminishes sharply near $110 \mathrm{~km}$ range. These data were recorded using a 128 baud, two microseconds baud length, complementary code. $a$ Indicates real part of the (decoded) complex signal from a single scan; $b$ indicates amplitude domain integration of the signal over $1000 \mathrm{~ms}(200$ scans); $c$ indicates difference $a-b$ 
modify the relative weighting factors of elements in the lag profile matrix and an additional processing stage may be required to correct for this.

The first step in clutter removal is to form an estimate of the clutter signal. In incoherent scatter experiments one can form a clutter profile estimate in the amplitude domain, for a given frequency and antenna direction, by adding together the measured complex-valued signals from several identical pulses.

If the individual signal measurements are retained in the memory, it is possible to subtract the clutter profile estimate from each individual signal data vector. Scaling against the true transmitted power may be needed for the best performance and this can be achieved at ESR where the individual transmitted pulses are routinely measured. The remaining signal processing continues as if the resulting data were normal incoherent scatter data uncontaminated by any clutter. A data example, using one second ESR integrations, illustrating how the integrated clutter profile subtraction works in practice is shown in Fig. 1.

Statistically, the method is good but after subtraction the residual noise represents a constant component in every data vector, creating correlations that weaken the statistical properties of the data and may be very harmful in some applications. The method can also lead to severe errors if the clutter exhibits significant amplitude and/or phase changes within the clutter profile accumulation time. Long clutter integration times are also difficult when using alternating codes because of the need to change the modulation pattern rapidly to complete the entire code sequence within the typical scale time of ionospheric changes. The method is thus most useful when using short integration times. The shortest possible integration time includes only two pulses and, in this important special case, a very simple computation algorithm, described in detail in the next section, can be used.

The method discussed requires processing in the amplitude domain. However, the clutter filtering can also be done in the power domain. The lag profile matrix (Turunen, 1986) is a linear combination of the temporal auto-correlation matrix of the wanted target signal, the temporal auto-correlation matrix of the bandwidth limited noise, and the spatial auto-correlation matrix of the possible ground clutter component of the signal. One can remove any of these three components by subtracting it from the sum providing that an estimate of the component is available.

An estimate of the white noise component is obtained by computing the matrix elements for bandwidth limited noise having the same true power, band pass properties and normalisation as the noise in the data matrix. The subsequent subtraction is often called background subtraction. The spatial auto-correlation matrix of the clutter can be obtained by using cross correlation matrix estimates computed from data vectors measured using identical modulations and having not too large separations in time. However, one has to consider the statistical properties of the process in more detail.
Any lag profile matrix element is based on the product of two samples. We assume here that the samples include a clutter component. It is enough to consider only one element in the matrix. Let the white noise + target scatter component be $x_{1}$ and $x_{2}$ at time instants $t_{1}$ and $t_{2}$ and let the corresponding superposed clutter components be $c_{1}$ and $c_{2}$. The required matrix element is:

$a_{12}=\left(x_{1}+c_{1}\right) \cdot\left(x_{2}+c_{2}\right)^{*}$

The product contains the following elements: $x_{1} x_{2}^{*}$, the wanted incoherent scatter target contribution, $c_{1} c_{2}^{*}$, elements of the spatial auto-correlation function of the clutter signal, and $x_{1} c_{2}^{*}+x_{2}^{*} c_{1}$, a zero-mean random process.

The third term is the product of the ground clutter signal and the randomly distributed numbers $x_{1}$ and $x_{2}$. The mean value of this term is zero, but it can create very high variance in $a_{12}$ because $c_{1}$ and $c_{2}$ can be very large. There is, however, a way to circumvent this. After one inter-pulse period, the corresponding samples contain the same clutter with values $c_{1}$ and $c_{2}$ but the target and noise contributions, say $y_{1}$ and $y_{2}$, are totally independent from $x_{1}$ and $x_{2}$. Repeating Eq. (1) and adding the two products together produces:

$$
\begin{aligned}
b_{12}= & \left(x_{1} x_{2}^{*}+y_{1} y_{2}^{*}\right)+2 c_{1} c_{2}^{*}+\left(x_{1}+y_{1}\right) c_{2}^{*} \\
& +\left(x_{2}+y_{2}\right)^{*} c_{1}
\end{aligned}
$$

The modified cross correlation matrix elements between these two vectors are:

$$
\begin{aligned}
d_{12}= & \left(x_{1} y_{2}^{*}+y_{1} x_{2}^{*}\right)+2 c_{1} c_{2}^{*}+\left(x_{1}+y_{1}\right) c_{2}^{*} \\
& +\left(x_{2}+y_{2}\right)^{*} c_{1}
\end{aligned}
$$

Subtracting Eq. (3) from Eq. (2) yields a difference, $e_{12}$ :

$e_{12}=b_{12}-d_{12}=\left(x_{1} x_{2}^{*}+y_{1} y_{2}^{*}\right)-\left(x_{1} y_{2}^{*}+y_{1} x_{2}^{*}\right)$

The first term $\left(x_{1} x_{2}^{*}+y_{1} y_{2}^{*}\right)$ contains the desired target contribution. Both the clutter component and the components causing the worst variance increase are totally removed. The remaining term, a sum of two products of independent numbers, increases the final variance such that the integration time needed to achieve a given accuracy increases by factor of two relative to the non-clutter filtered case. The result is identical to the integrated clutter profile method described earlier for cases where only two points are used to form the clutter profile estimate. The power domain solution requires more real time computations than the amplitude domain process and seems to offer no real benefits over the pulse-to-pulse subtraction method described later.

\section{The pulse-to-pulse subtraction method}

A special case of the integrated clutter profile subtraction method is when the integration is performed over only two identical pulses. A simple computation algorithm can now be used in which data vectors are subtracted point by point in the amplitude domain. The principles of the method are illustrated graphically in Fig. 2. 


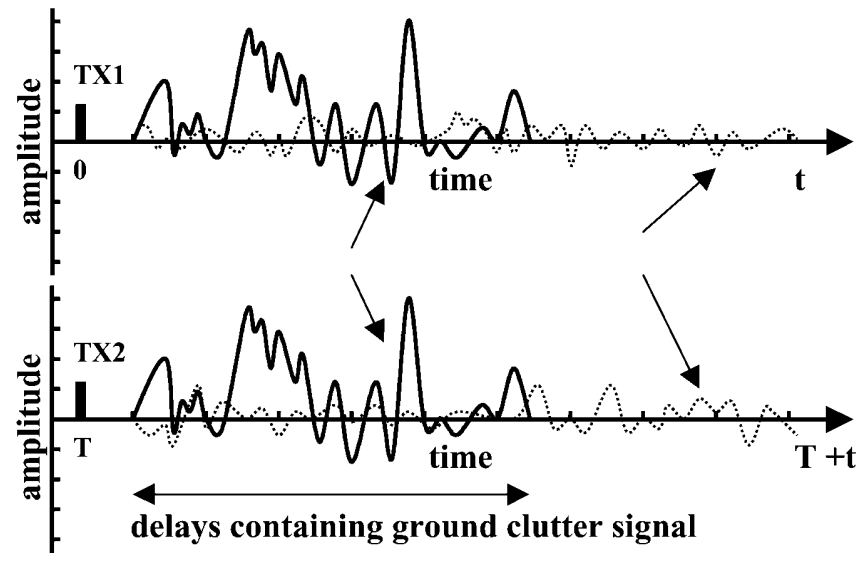

Fig. 2. Schematic illustration of the principles of the pulse-to-pulse subtraction method. Identical pulses TX1 and TX2 are transmitted at instants $t=0$ and $t=T$ and the returned signal is received as a function of the delay time $t$. Providing that $T$ is both much shorter than the coherence time of the ground clutter signal and much longer than the coherence time of the target signal, the ground clutter amplitudes are identical and the wanted signal + noise components are independent. For simplicity only one of the complex components (real or imaginary) of the return signal is shown here. The ground clutter signal and the target signal + noise have been drawn separately although in reality, of course, the sum of the two signals is received. When the signals received at $t$ and $T+t$ are subtracted, the ground clutter components exactly cancel each other. The remaining difference signal is statistically identical to the incoherent scatter signal that would be obtained in a clutter free environment. The amount of data is halved, which increases the required integration time by a factor of two

As before, let the white noise + target scatter components be $x_{1}$ and $x_{2}$ at time instants $t_{1}$ and $t_{2}$ and let the corresponding superposed clutter components be $c_{1}$ and $c_{2}$. Some tens of milliseconds later the corresponding samples still contain the same clutter, $c_{1}$ and $c_{2}$, but the target and noise contributions, $y_{1}$ and $y_{2}$, are totally independent from $x_{1}$ and $x_{2}$. Subtracting the two sample sets gives a new set of elements $z_{1}$ and $z_{2}$ :

$z_{1}=\left(x_{1}+c_{1}\right)-\left(y_{1}+c_{1}\right)=x_{1}-y_{1}$

$z_{2}=\left(x_{2}+c_{2}\right)-\left(y_{2}+c_{2}\right)=x_{2}-y_{2}$

These data can be treated as clutter free incoherent scatter data and a lag profile matrix element can be computed using $z_{1}$ and $z_{2}$ :

$a_{12}=z_{1} \cdot z_{2}^{*}=\left(x_{1} x_{2}^{*}+y_{1} y_{2}^{*}\right)-\left(x_{1} y_{2}^{*}+y_{1} x_{2}^{*}\right)$

It should be noted that Eqs. (4) and (7) are identical. This method has been used at the EISCAT Svalbard radar since August 1998. In general, the two data vectors should be scaled for transmitter power variations before subtraction, but, at the ESR, successive pulses are sufficiently similar that this is unnecessary. In practical experiments individual pulses within adjacent pulse groups are different and the closest available pairs of identical pulses must be selected, even when these are not drawn from the two closest transmitted pulses.

The modulation used at short ranges, where ground clutter filtering must be applied, is usually an alternating code. The same transmitted pulse works well, and will generally also be used, at much greater ranges and only

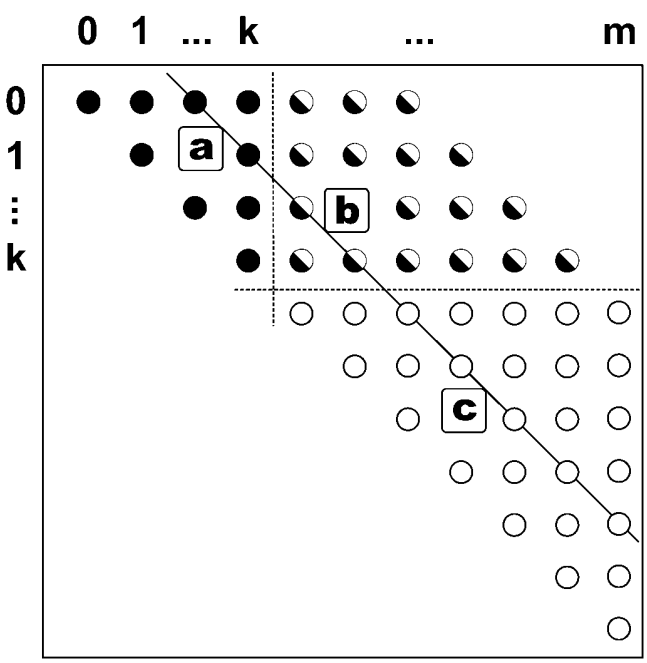

Fig. 3. Schematic illustration of different types of lag profile matrix elements appearing when the first $k+1$ samples in the data vector having $m+1$ samples have been ground clutter filtered by pulse-topulse subtraction before matrix computation. a Solid circles represent elements with both samples filtered. The variance is doubled and the weighting factor is two. $b$ Half solid circles represent elements for which only one sample in the product is filtered. The variance is doubled and the weighting factor is one. c Open circles represent matrix elements with no clutter filtering. The weighting factor correction must be applied before applying any FIR filters required for decoding alternating codes

relatively small parts of the total range covered will therefore need ground clutter filtering. The simplest approach is to ignore this and perform the filtering for all the ranges but this is not very efficient. A better way is to form two separate data vectors from the same original data, one filtered to cover the lowest ranges and the other unfiltered and computed separately.

However, it is more effective to compute directly over the boundary producing three different kinds of elements in the lag profile matrix as shown schematically in Fig. 3. Every element of the lag profile matrix is a product of two data samples and, close to the boundary region, one, both or neither of these will be filtered. Assume that two complex data vectors, $x_{i}$ and $y_{i}, i=0$, $m$, have been sampled from two separate illuminations of the target and that the first $k+1$ elements $x_{i}$ and $y_{j}$ include a clutter component. A new clutter-subtracted vector can be formed:

$z_{i}=x_{i}-y_{i} \quad i=0, k$

and the original data vectors replaced by:

$u_{i}= \begin{cases}z_{i} & i=0, k \\ x_{i} & i=k+1, m\end{cases}$
$v_{i}= \begin{cases}-z_{i} & i=0, k \\ y_{i} & i=k+1, m\end{cases}$

The lag profile matrix is now computed using the new data vectors $u_{i}$ and $v_{i}$. If both of the elements are filtered, the lag product $a_{i j}$ at lag $j$ is of the form:

$$
a_{i j}=u_{i} \cdot u_{i+j}^{*}=z_{i} \cdot z_{i+j}^{*}=x_{i} x_{i+j}^{*}+y_{i} y_{i+j}^{*}-x_{i} y_{i+j}^{*}-y_{i} x_{i+j}^{*}
$$


The first two terms are the wanted terms with non-zero expectation value and the last two terms, which have zero expectation values, only increase the variance compared with the unfiltered data. The weighting factor for these terms, compared with the rest of the data in Eqs. (9) and (10), is two if the computations are done identically over the whole set, because the same terms appear twice, and the integration time needed for a given accuracy is doubled.

If only one element is clutter filtered, the lag product is of the form:

$a_{i j}=u_{i} \cdot u_{i+j}^{*}=z_{i} \cdot x_{i+j}^{*}=x_{i} x_{i+j}^{*}-y_{i} x_{i+j}^{*}$

The first term is the wanted term, while the second has zero mean but gives extra variance and again doubles the necessary integration time for a given accuracy. The weighting factor is 1 for these terms.

Finally, where neither element is clutter subtracted, the integration time and weighting factors are both unity and the lag product is of the form:

$a_{i j}=u_{i} \cdot u_{i+j}^{*}=x_{i} x_{i+j}^{*}$

The lowest part of the target is often measured by using alternating codes. The bias in the product expectation values due to the different weighting factors can be corrected at various points in the computation but, in order to minimise the variance of the data, it should be removed (by dividing by 2 as indicated) before the FIR filtering needed to decode the alternating codes.

The method can be used for all modulations provided only that the same modulation pattern appears at least twice in the transmission sequence and that the time difference between these two transmissions is sufficiently short. The integration time is necessarily doubled, and so also is the target stationarity requirement because the same modulation has to be sent twice, but this is not an unreasonably high price. It can also be expanded to cover D-layer experiments, though this has not yet been tested at the ESR, but the exact response of the clutter filtering in the frequency domain must be carefully considered. In normal incoherent scatter applications this has no practical effect.

An example showing analysed ionospheric parameters using ESR incoherent scatter data is shown in Fig. 4. Filtering by pulse-to-pulse subtraction has totally eliminated the original strong clutter contamination and allows previously hidden E-region detail to be studied, see Fig. 5.

This method is very effective when the cluttering signals arise from stationary or slowly moving targets. With the parameters employed at the ESR, clutter arising from targets moving with line-of-sight velocities greater than about $1 \mathrm{~ms}^{-1}$ would not be well suppressed. However, such targets are virtually absent from the local clutter environment, which is completely dominated by hard target reflections form bare mountains, and such disturbances are insignificant in practice.

\section{Conclusions}

The pulse-to-pulse subtraction method has been used at the EISCAT Svalbard Radar since August 1998 and has been shown to be a practical and reliable method for removing ground clutter from the received signals. Other possible techniques include subtraction of an integrated clutter profile in the amplitude domain and subtraction of a clutter profile estimate obtained using gup3 13-Aug-1998 19:50:00-19:52:00 AVE 180.6/81.6 0.97 MW
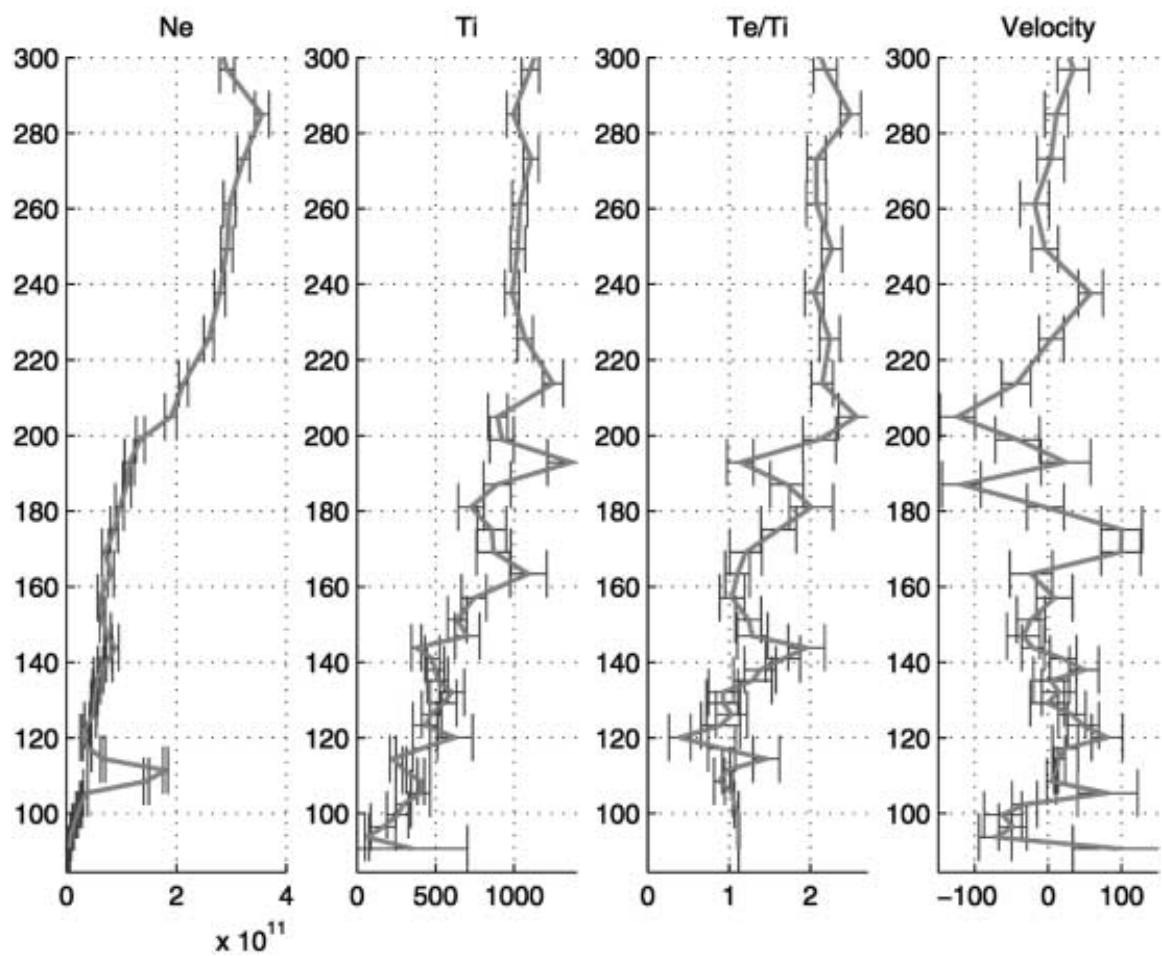

Fig. 4. Examples of ionospheric profiles, with error bars, of electron density, ion temperature, the ratio of electron to ion temperature and the line of sight ion velocity (positive away from the radar) obtained by the ESR after applying ground clutter filtering 
Longyearbyen 13-Aug-1998 Az 181 El 82
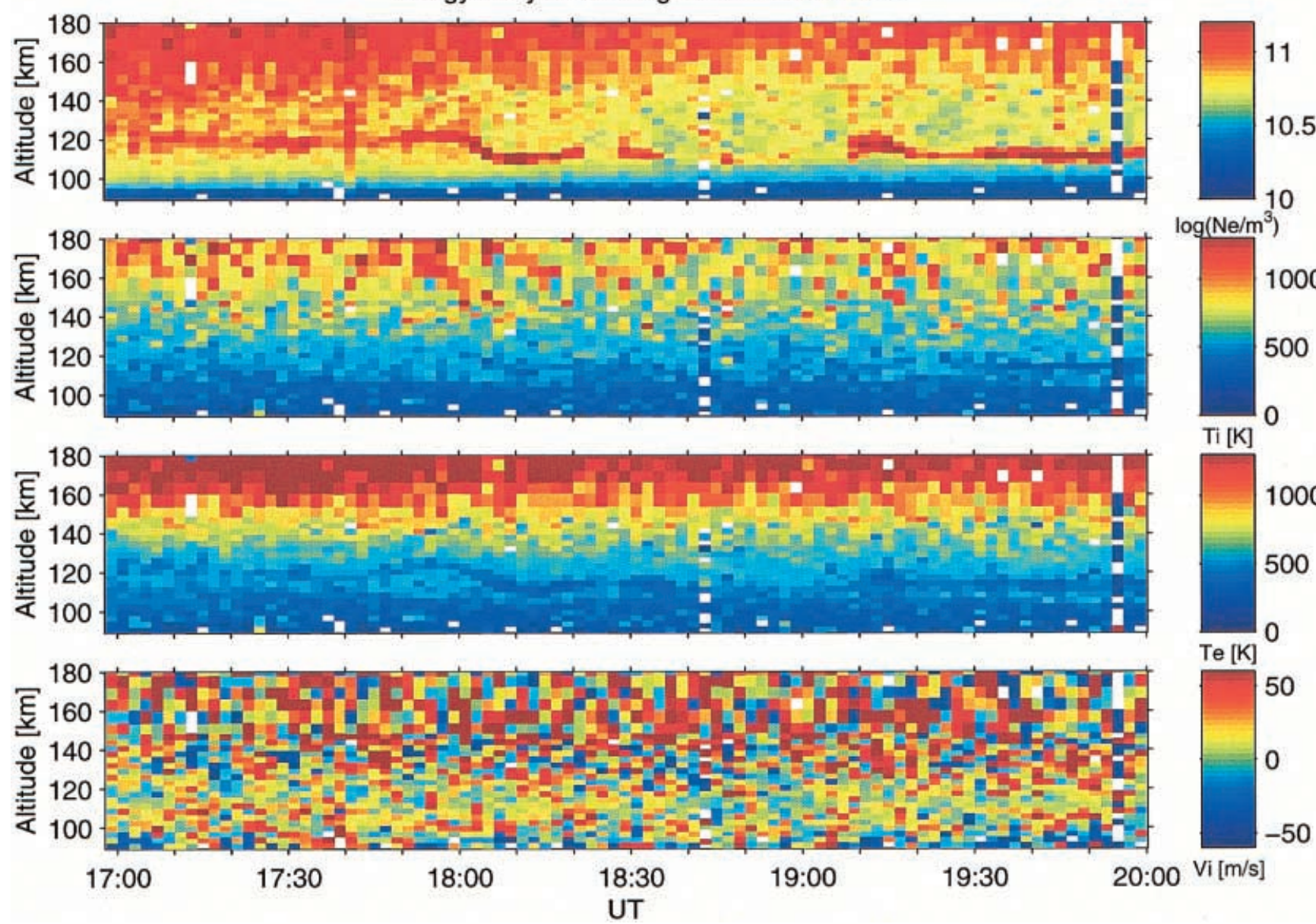

Fig. 5. Three hours of fitted ionospheric parameters (as Fig. 4) obtained using EISCAT Svalbard Radar data under strong clutter conditions. The clear Sporadic-E layer illustrates the success of the clutter removal procedure. It should be noted that the negative

modified cross correlations in the power domain. For the special case in which only two data vectors are used, all three methods have been shown to be identical.

In this discussion, it was assumed that the amplitude and phase of the clutter remained constant over the required integration period. However, it is possible to relax that assumption. Linear amplitude and phase changes in the cluttering signal at a given range as a function of time could be considered, and more complicated approaches are also possible, but the practical limitation is the available real time computation power. In all cases, ground clutter filtering effects only the lowest parts of the target where it increases the integration time required for a given accuracy by a factor of two. More sophisticated approaches cannot gain more than this factor and, for normal applications, the simple pulse-topulse subtraction method is good enough.

Acknowledgements. The EISCAT Scientific Association is supported by the Centre National de la recherche Scientifique (France), the Max-Planck Gesellschaft (Germany), the National Institute of Polar Research (Japan), Naturvetenskapliga Forskningsrdet (Sweden), the Particle Physics and Astronomy Research Council (United Kingdom), the Research Council of Norway (Norway) and Suomen Akatemia (Finland). excursions in temperature associated with this layer are an artefact of the analysis procedure which did not fit for the changes in ion composition in such layers

Topical Editor M. Lester thanks C.J. Heinselman and I. McCrea for their help in evaluating this paper.

\section{References}

Folkestad, K., T. Hagfors, and S. Westerlund, EISCAT: An updated description of technical characteristics and operational capabilities, Radio Sci., 18, 867-879, 1983.

Hagfors, T., Plasma fluctuations excited by charged particle motion and their detection by weak scattering of radio waves, in Incoherent scatter, theory, practice and Science, Ed. De Alcaydé, EISCAT Technical Report 97/53, 1997.

Lehtinen, M. S., and I. Häggström, A new modulation principle for incoherent scatter measurements, Radio Sci., 22, 625-634, 1987.

Turunen, T., and $\mathbf{J}$. Silén, Modulation patterns for the EISCAT incoherent scatter radar, J. Atmos. Terr. Phys., 46, 593-599, 1984.

Turunen, T., GEN-SYSTEM a new experimental philosophy for EISCAT radars, J. Atmos. Terr. Phys., 48, 777-785, 1986.

Wannberg, G., I. Wolf, L.-G. Vanhainen, K. Koskenniemi, J. Röttger, M. Postila, J. Markkanen, R. Jacobsen, A. Stenberg, R. Larsen, S. Eliassen, S. Heck, and A. Huuskonen, The EISCAT Svalbard radar: a case study in modern incoherent scatter radar system design, Radio Sci., 32, (6) 2283-2307, 1997.

Reference is also made to the following unpublished material:

EISCAT Annual Report 1996-1997, EISCAT Headquarters, 1997. 